Article

\title{
Beneficial Effects of Lactobacillus plantarum Strains on Non-Alcoholic Fatty Liver Disease in High Fat/High Fructose Diet-Fed Rats
}

\author{
Eun-Jung Park ${ }^{1,2,+} \mathbb{D}$, You-Suk Lee ${ }^{1,2,+}$, Sung Min Kim ${ }^{1}$, Gun-Seok Park ${ }^{3}$, Yong Hyun Lee ${ }^{3}$, \\ Do Yeun Jeong ${ }^{3}$, Jihee Kang ${ }^{3}$ and Hae-Jeung Lee ${ }^{1,2, *(1)}$ \\ 1 Department of Food and Nutrition, Gachon University, Seongnam, Gyeonggi-do 13120, Korea; \\ ejpark@gachon.ac.kr (E.-J.P.); ysleeyun@gachon.ac.kr (Y.-S.L.); kimsm127@gc.gachon.ac.kr (S.M.K.) \\ 2 Institute for Aging and Clinical Nutrition Research, Gachon University, Seongnam, \\ Gyeonggi-do 13120, Korea \\ 3 AtoGen Co., Ltd., Daejeon, 34015, Korea; gspark@atogen.co.kr (G.-S.P.); tppoc002@atogen.co.kr (Y.H.L.); \\ dyjeong@atogen.co.kr (D.-Y.J.); jhkang@atogen.co.kr (J.K.) \\ * Correspondence: skysea@gachon.ac.kr; Tel.: +82-31-750-5968 \\ + These authors contributed equally to this work.
}

Received: 30 January 2020; Accepted: 17 February 2020; Published: 20 February 2020

\begin{abstract}
Emerging evidence suggests that probiotics are beneficial in non-alcoholic fatty liver disease (NAFLD). This study aimed to explore the effects of two Lactobacillus plantarum strains, ATG-K2 and ATG-K6 (isolated from Korean fermented cabbage), in a rat model of high fat/high fructose (HF/HF) diet-induced NAFLD. Rats with NAFLD were randomized into four groups (HF/HF diet control, (HC); HF/HF diet with silymarin, (PC); HF/HF diet with ATG-K2, (K2); and HF/HF diet with ATG-K6, (K6)) with healthy rats on a normal diet serving as the negative control. After treatment, histopathological and biochemical analyses of the blood and liver tissue were conducted. In addition, fecal microbiota was analyzed using the MiSeq platform. Compared with HC rats, K2 and K6 rats experienced significantly lower body weight gain, displayed decreased hepatic lipid accumulation, had lower serum levels of aspartate aminotransferase and alanine aminotransferase, and showed increased antioxidant enzyme activities. Moreover, de novo lipogenesis-related genes were downregulated following $\mathrm{K} 2$ and $\mathrm{K} 6$ administration. The fecal microbiota of $\mathrm{K} 2$ and $\mathrm{K} 6$ rats contained a higher proportion of Bacteriodetes and a lower proportion of Fimicutes than that of HC rats. Taken together, our results suggest that L. plantarum strains ATG-K2 and ATG-K6 are potential therapeutic agents for NAFLD.
\end{abstract}

Keywords: Lactobacillus plantarum; high-fat/high-fructose diet; non-alcoholic fatty liver disease

\section{Introduction}

The increase in non-communicable metabolic diseases, such as chronic liver disease, type 2 diabetes, and heart disease, is linked to rising obesity rates [1]. Approximately $90 \%$ of patients with severe obesity are diagnosed with non-alcoholic fatty liver disease (NAFLD), 37\% with non-alcoholic fatty hepatitis, and 10\% with liver cirrhosis [2]. NAFLD, which is characterized by lipid accumulation in over $5 \%$ of hepatocytes in the absence of excessive alcohol consumption, is one of the most prevalent liver diseases [3,4]. The condition does not usually cause any symptoms in its early stage, but it can progress to serious liver diseases, including liver fibrosis, cirrhosis, and cancer [5].

Sugars, especially fructose, can activate hepatic lipogenesis programs that exacerbate NAFLD [6]. Fructose is processed almost exclusively by the liver, where it is mostly metabolized to triglycerides (TG) by de novo lipogenesis (DNL) [6,7]. Fructose-derived precursors act as nutritional regulators 
of the transcription factors, such as sterol regulatory element binding protein 1c (SREBP-1c) and $\mathrm{CCAAT} /$ enhancer-binding protein alpha $(\mathrm{C} / \mathrm{EBP} \alpha)$, which in turn regulate the expression of hepatic gluconeogenesis and DNL genes [6]. It follows that fructose intake increases liver gluconeogenesis and DNL and elevates blood glucose and TG levels in humans [8,9]

Another cause of NAFLD is oxidative stress. Fat deposition occurring in the liver results in lipid peroxidation, and it promotes a variety of responses such as inflammation and fibrosis. Thus, antioxidants (e.g., silymarin, vitamin E) are proposed as candidates for NAFLD treatment $[10,11]$; however, there are no standard pharmacological therapeutic agents for NAFLD yet. Recently, emerging evidence suggests that the gut-liver axis is strongly related to NAFLD. There are several reports published clinical trials applying probiotics on NAFLD patients [12]. Lactobacillus bulgaricus and Streptococcus thermophiles treatment improved liver aminotransferases levels in adult NAFLD patients [13]. In addition, probiotic capsules containing Lactobacillus acidophilus, Bifidobacterium lactis, Bifidobacterium bifidum, and Lactobacillus rhamnosus, as a supplement also decreased liver aminotransferases levels in obese children with NAFLD [14]. These results indicate that the intake of certain probiotics has a beneficial effect on NAFLD.

Lactobacillus plantarum, which plays a key role in the production of various fermented foods, is one of the most important species of lactic acid bacteria (LAB). L. plantarum is found in diverse environments, such as the soil and the human gut, and is considered a potential probiotic [15]. In addition, some strains of $L$. plantarum exert hepatoprotective effects by improving liver enzyme activity and reducing serum TG and total cholesterol (TC) levels [16,17]; however, the role of L. plantarum in NAFLD is still unclear.

Two novel L. plantarum strains, ATG-K2 (K2) and ATG-K6 (K6), have been recently isolated from fermented cabbage, a Korean traditional food [18]. $\mathrm{K} 2$ and $\mathrm{K} 6$ display bile salt hydrolase (BSH) activity, an ability to generate hydrogen peroxide $\left(\mathrm{H}_{2} \mathrm{O}_{2}\right)$, immunomodulatory effects, and antimicrobial properties [18]. BSH activity is important for secondary bile salt metabolism, which may contribute to bile resistance of bacteria and benefit the host's cholesterol metabolism by lowering bile salt concentration [19]. $\mathrm{H}_{2} \mathrm{O}_{2}$ produced by L. plantarum strains may help to kill opportunistic pathogens and have radical scavenging activity [20]. This indicates that $\mathrm{K} 2$ and $\mathrm{K} 6$ could be attractive biological agents for antimicrobial. Many supplements with antioxidant and anti-inflammatory properties are also effective in NAFLD. Thus, we hypothesized that K2 and K6 would have the potential to ameliorate NAFLD. In this study, we aimed to assess the effects of L. plantarum K2 and K6 on NAFLD using an in vivo rat model.

\section{Materials and Methods}

\subsection{Preparation of L. plantarum Strains}

The K2 and K6 L. plantarum strains were isolated from Korean fermented cabbage as part of a previous study [18] and deposited in Korean Collection for Type Cultures (KCTC; https://kctc.kribb.re. $\mathrm{kr} / \mathrm{En} / \mathrm{Kctc}$.aspx). Based on average nucleotide identity analysis [21], K2 and K6 showed $96.6 \%$ and $99.16 \%$ of sequence identity with type strain ATCC 14917. Details of the general features of L. plantarum strains K2 and K6 are shown in Table S1. The strains were cultured in de Man-Rogosa-Sharpe (MRS) medium (Difco Laboratories, Detroit, MI, USA) at $37^{\circ} \mathrm{C}$ for $16 \mathrm{~h}$. Subsequently, K2 and $\mathrm{K} 6$ were collected by centrifugation at $8000 \mathrm{rpm}$ for $15 \mathrm{~min}$ at $4{ }^{\circ} \mathrm{C}$ and washed three times with phosphate buffered saline (PBS). Finally, the cells were suspended in 2 volumes of $12.5 \%$ trehalose, $10 \%$ skim milk, and $0.125 \%$ carboxymethyl cellulose (w/v) solution and lyophilized using a FD8508 freeze-dryer (IlShinBioBase, Dongduchen, Korea). The colony-forming units (cfu) of dried K2 and K6 powder were quantified by count on MRS-agar plate. The freeze-dried powder was re-suspended in PBS before it was orally administered to rats. 


\subsection{Animals and Treatment}

A total of 42 male Wistar rats were purchased from Orient Bio Co. Ltd. (Seongnam, Korea) and housed under a controlled temperature $\left(20-25^{\circ} \mathrm{C}\right)$ and humidity $(50-55 \%)$, with a $12 \mathrm{~h} / 12 \mathrm{~h}$ light-dark cycle. Figure 1 shows schematic summary of the experimental schedule. The rats were allowed to acclimatize for seven days before they were randomly divided into two groups: healthy control group and NAFLD group. The rats were given free access to food and water, and were fed either a normal diet (D12450B; Research Diets, NJ, USA) or a high-fat (45\%) diet (D12451, Research diet, NJ, USA) with $10 \%$ fructose in the drinking water $(\mathrm{HF} / \mathrm{HF})$. After eight weeks, one rat from each group was sacrificed to assess for the presence of fatty liver. Following NAFLD induction, the rats were randomized into five groups ( $n=8$ per group) according to their body weight: normal diet control (NC), $\mathrm{HF} / \mathrm{HF}$ diet control (HC), HF/HF diet with $100 \mathrm{mg} / \mathrm{kg}$ silymarin (positive control, PC), HF/HF diet with $\mathrm{K} 2$ (5 $\left.\times 10^{8} \mathrm{cfu}, \mathrm{K} 2\right)$, and $\mathrm{HF} / \mathrm{HF}$ diet with $\mathrm{K} 6\left(5 \times 10^{8} \mathrm{cfu}, \mathrm{K} 6\right) . \mathrm{NC}$ and $\mathrm{HC}$ rats were administered PBS, while $\mathrm{PC}, \mathrm{K} 2$, and $\mathrm{K} 6$ rats were treated with the indicated materials using a sonde for an additional 8 weeks. Subsequently, all rats were fasted overnight and weighed before they were euthanized. Blood was collected by cardiac puncture. The blood was then centrifuged at $3000 \mathrm{rpm}$ for $15 \mathrm{~min}$ at $4{ }^{\circ} \mathrm{C}$ (Combi-514R, Hanil Co. Ltd., Seoul, Korea) to extract the serum, which was stored at $-80{ }^{\circ} \mathrm{C}$ until analysis. The rat livers were removed, rinsed with PBS, and stored immediately at $-80{ }^{\circ} \mathrm{C}$ for future use. All animal procedures were approved by Gachon University for the care and use of the laboratory animals (ref. no. GIAUAC -R2019014).

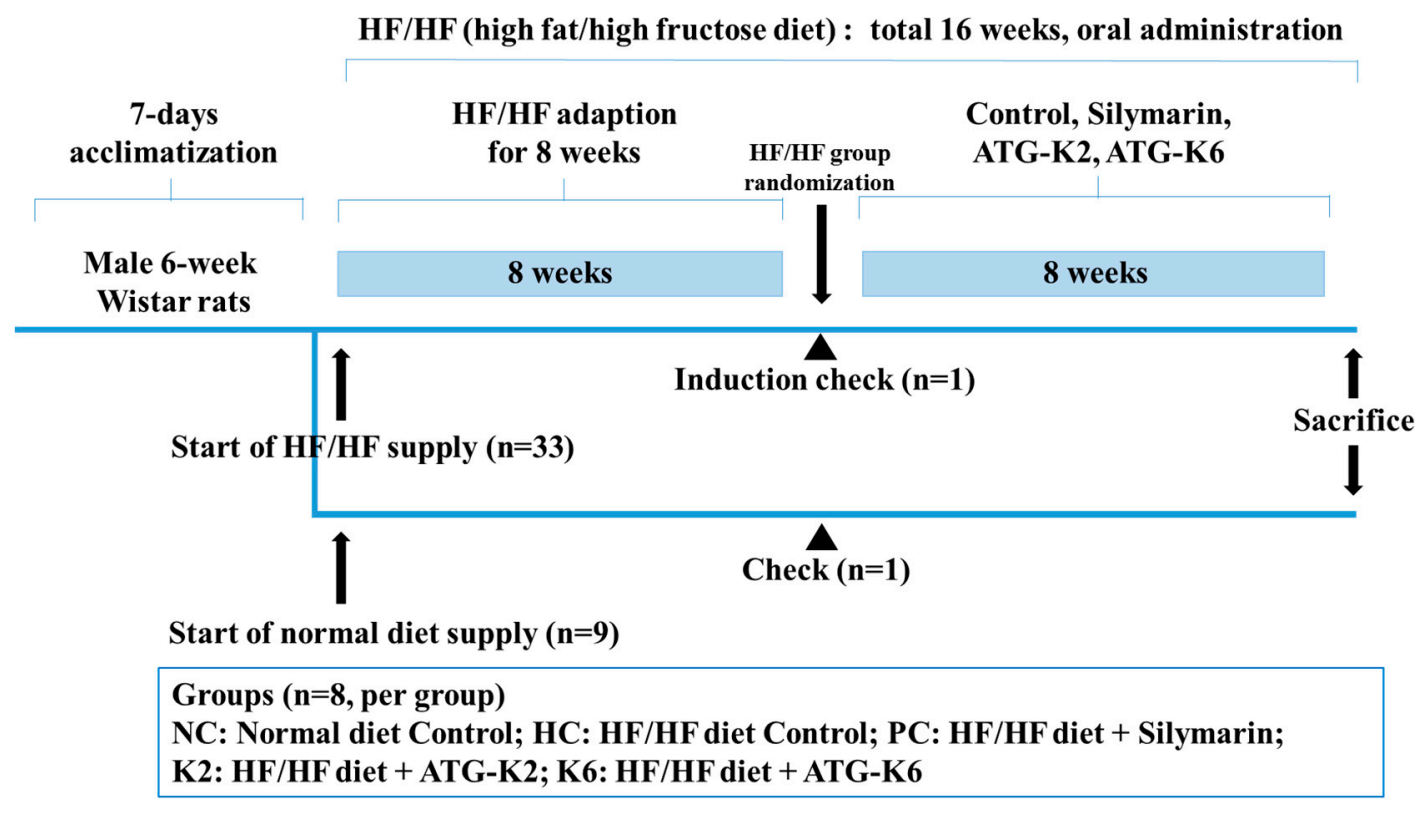

Figure 1. Schematic representation of the experimental schedule. HF/HF, high fat/high fructose.

\subsection{Histological Analysis and Oil-Red O Staining}

Samples of liver and epididymal tissue were fixed in 10\% formalin (Sigma-Aldrich, St. Louis, MO, USA), embedded in paraffin wax, processed into 3-4 $\mu \mathrm{m}$ sections, and stained with hematoxylin and eosin (H\&E). Gun-sucrose-fixed liver sections were stained with Oil-red $\mathrm{O}$ and observed under an Olympus Provis AX70 microscope (Olympus, Tokyo, Japan); images were captured with a Nikon DS-Ri2 camera (Nikon, Tokyo, Japan) using NIS-Elements BR 4.50.00 software (Tokyo, Japan).

\subsection{Measurement of Serum Biochemistry Markers}

Serum alanine aminotransferase (ALT), aspartate aminotransferase (AST), and alkaline phosphatase (ALP) levels were measured using ALT, AST, and ALP assay kits (Asanpharm, Hwaseong, 
Korea). Serum TG, TC, and high-density lipoprotein cholesterol (HDL-C) levels were quantified using TG, T-CHO, and HDL-CHO kits (Asanpharm, Hwaseong, Korea), respectively. Serum glucose levels were determined using a blood glucose meter kit (Handok, Seoul, Korea). Serum adiponectin and leptin levels were measured by an enzyme-linked immunosorbent assay (ELISA) using the appropriate kits (R\&D system, Minneapolis, MN, USA). Colorimetric assay kits (BlueGene Biotech, Shanghai, China) were used to quantify serum levels of superoxide dismutase (SOD), glutathione peroxidase (GPx), and catalase (CAT). Data were calculated using the formulae described in each kit.

\subsection{Measurement of Liver Biochemistry Markers}

Hepatic samples were extracted according to the method of Folch, with some modifications. Liver tissue $(0.1 \mathrm{~g})$ was homogenized in a chloroform/methanol $(2: 1, \mathrm{v} / \mathrm{v})$ solution, vortexed, and then centrifuged at $3000 \mathrm{rpm}$ for $10 \mathrm{~min}$ at room temperature. After centrifugation, the bottom layer was carefully aspirated into a fresh test tube and evaporated to dryness under a stream of nitrogen. The dried lipid layer was weighed and re-dissolved in methanol prior to analysis. Hepatic TG and TC content was measured using the TG and T-CHO kits (Asanpharm, Hwaseong, Korea).

Malondialdehyde (MDA) levels were measured as previously described [22]. Briefly, MDA levels were quantified in the clear supernatant by measuring the absorbance at $532 \mathrm{~nm}$, and expressed as $\mathrm{nmol} / \mathrm{g}$ of liver tissue, with 1,1,3,3-tetraethoxypropane (Sigma-Aldrich, St. Louis, MO, USA) used as the external standard.

\subsection{Quantitative Reverse Transcription PCR ( $q R T-P C R$ )}

Total RNA was extracted from homogenized liver tissue using the easy-spin ${ }^{\mathrm{TM}}$ total RNA extraction kit (iNtRON Biotechnology, seongnam, Korea). RNA (50 ng) was converted to cDNA using the GoScript ${ }^{\mathrm{TM}}$ Reverse Transcriptase (Promega, Madison, WI, USA) and used as a template for real-time RT-PCR analysis with SYBR Green Master Mix (TaKaRa Bio, Otsu, Japan) using ABI QuantStudio 3 (Applied Biosystems, Foster City, CA, USA). Details of the primers used in the study are shown in Table 1. Target gene expression was normalized to that of Actb ( $\beta$-actin).

Table 1. Primer sequences used for qRT-PCR.

\begin{tabular}{ccc}
\hline Gene & Forward $\left(\mathbf{5}^{\prime} \mathbf{-} \mathbf{3}^{\prime} \mathbf{)}\right.$ & Reverse $\mathbf{( 5}^{\prime} \mathbf{-} \mathbf{3}^{\prime} \mathbf{)}$ \\
\hline SREBP-1C & CCC TGC GAA GTG CTC ACA A & GCG TTT CTA CCA CTT CAG GTT TCA \\
FAS & GCT GCT ACA AAC AGG ACC ATC AC & TCT TGC TGG CCT CCA CTG AC \\
C/EBP $\alpha$ & GCC AAG AAG TCG GTG GAT AA & CCT TGA CCA AGG AGC TCT CA \\
ACC & CAA TCC TCG GCA CAT GGA GA & GCT CAG CCA AGC GGA TGT AGA \\
CPT-1 & CCA TCT CTT CTG CCT CTA TGT & GTC AGG GTT TTT CTC AAA GTC \\
$\beta$-actin & GAT TAC TGC CCT GGC TCC TA & TCA TCG TAC TCC TGC TTG CT
\end{tabular}

SREBP-1c, Sterol regulatory element binding protein 1c; FAS, Fatty acid synthase; C/EBP $\alpha$, CCAAT/enhancer-binding protein alpha; ACC, Acetyl-CoA carboxylase; CPT-1, Carnitine palmitoyltransferase-1.

\subsection{Protein Extraction and Western Blotting}

Proteins were extracted from the liver tissue samples using PRO-PREPTM Protein Extraction Solution (iNtRON Biotechnology, seongnam, Korea) containing the Halt ${ }^{\mathrm{TM}}$ phosphatase inhibitor cocktail (Thermo Scientific, Waltham, MA, USA). Following quantification with the Bicinchoninic acid assay (Thermo Scientific, Waltham, MA, USA), protein samples (30 $\mu \mathrm{g}$ ) were separated by $6-10 \%$ sodium dodecyl sulfate polyacrylamide gel electrophoresis, then transferred onto polyvinylidene fluoride membranes. The membranes were blocked with $5 \%$ skim milk for $1 \mathrm{~h}$ at room temperature, and then washed and incubated with the indicated antibodies. Antibodies against SREBP-1c, fatty acid synthase (FAS), and CCAAT/enhancer-binding protein alpha $(\mathrm{C} / \mathrm{EBP} \alpha)$ were purchased from Santa Cruz Biotechnology (California, CA, USA). Antibodies against AMPK and phospho-AMPK (Thr172) were purchased from Cell Signaling Technology (Danvers, MA, USA). Antibodies against acetyl-CoA 
carboxylase (ACC), phospho-ACC (Ser79), carnitine palmitoyltransferase-1 (CPT1), and $\beta$-actin were obtained from Abcam (Cambridge, MA, USA). Horseradish peroxidase (HRP)-conjugated secondary antibodies were purchased from Promega. The signals were detected using the WEST-Queen ${ }^{\mathrm{TM}}$ RTS Western Blot Detection Kit (iNtRON Biotechnology, seongnam, Korea); the immunoreactive bands were visualized by chemiluminescence using the ImageQuant LAS 500 imager (GE Healthcare Life Sciences, Little Chalfont, UK).

\subsection{Fecal Microbiota Profiling}

Stool samples were collected from the rats at termination, using sterile tweezers. The metagenomic DNA was extracted using the QIAamp PowerFecal DNA Kit (QIAGEN, Germany). The quantity and quality of extracted DNA were measured using the Qubit 4 Fluorometer (Thermo Scientific, Waltham, MA, USA), NanoDrop One Microvolume UV-Vis Spectrophotometer (Thermo Scientific, Waltham, MA, USA), and agarose gel electrophoresis. The V3-V4 hypervariable regions of the bacterial $16 \mathrm{~S}$ rRNA were amplified with unique $8 \mathrm{bp}$ barcodes and sequenced on the Illumina MiSeq PE300 platform according to standard protocol [23]. The sequenced reads were analyzed using the Quantitative Insights into Microbial Ecology (QIIME) pipeline [24]. Briefly, sequences were filtered and clustered into operational taxonomic units (OTUs) at 97\% sequence identity according to the SILVA 128 database [25] before weighted UniFrac distances matrices were calculated for principal coordination analysis (PCoA) [26].

\subsection{Statistical Analysis}

The data were expressed as mean \pm standard deviation ( $n=8$ per group). One-way analysis of variance (ANOVA) with Duncan's multiple range tests was used to perform multiple comparisons between the groups. All statistical analyses were carried out in SPSS version 25 (SPSS Inc., Chicago, IL, USA). $p$-values of $<0.05$ were considered statistically significant.

\section{Results}

3.1. Effect of the L. plantarum K2 and K6 on Hepatic Steatosis and Body Weight in the Rat Model of HF/HF Diet-Induced NAFLD

We first assessed hepatic steatosis in the rat model. Visual inspection of rat liver tissue following eight weeks of a HF/HF diet revealed altered color and lipid accumulation. To investigate the effect of $L$. plantarum $\mathrm{K} 2$ and $\mathrm{K} 6$ on hepatic fat accumulation, we assessed hepatic steatosis by visual examination, $\mathrm{H} \& \mathrm{E}$ staining, and Oil-red O staining after eight weeks of treatment (Figure 2a). H\&E staining showed widespread lipid deposits in the HC group, with less deposits in the PC, K2, and K6 groups (Figure 2b). Oil Red O staining confirmed these findings. Moreover, H\&E staining of epididymal fat tissue revealed that adipocyte size was smaller in the $\mathrm{K} 2$ and $\mathrm{K} 6$ groups than in the $\mathrm{HC}$ group.

As shown in Figure 2c and Table 2, final body weights of rats in the HC group were higher than those of rats in the NC group. In addition, higher weight gain was observed in HC rats than in NC rats. In contrast to $\mathrm{HC}$ feeding, $\mathrm{K} 2$ and $\mathrm{K} 6$ administration significantly suppressed body weight gain as well as PC groups. 
(a)

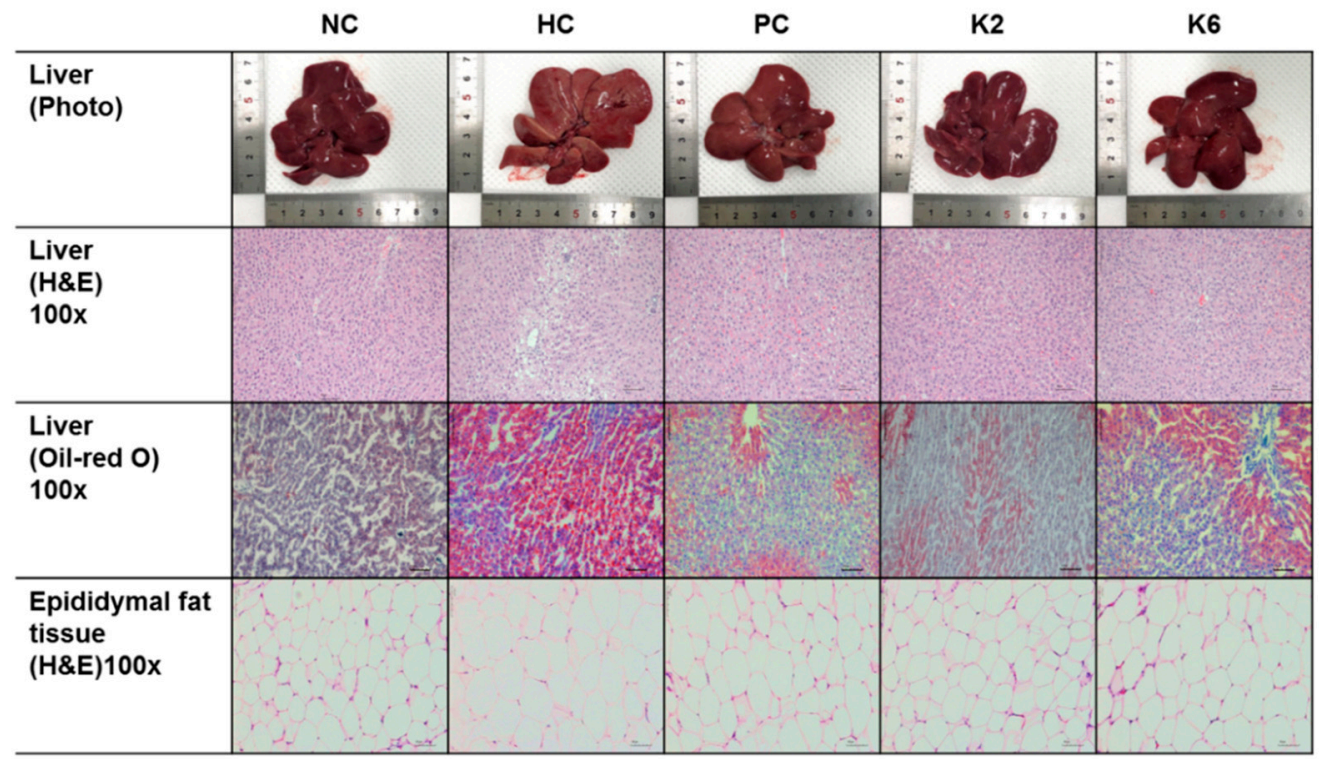

(b)

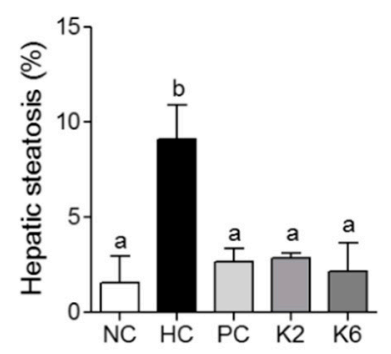

(c)

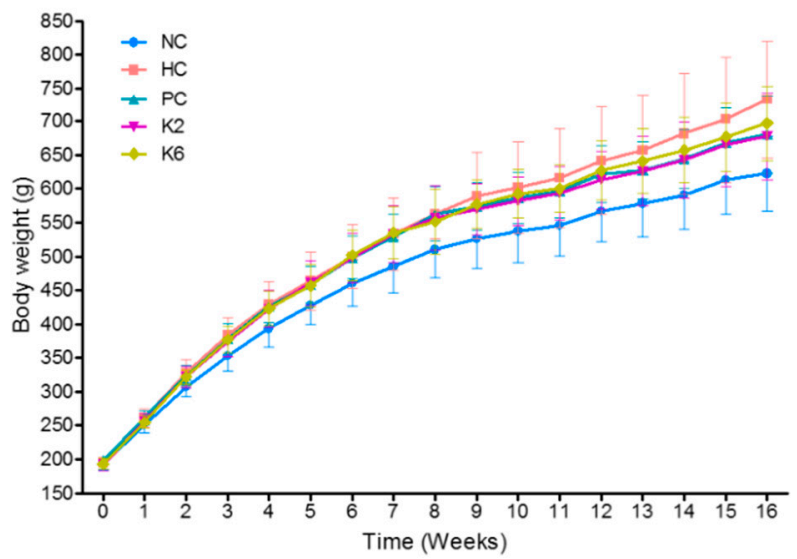

Figure 2. Effects of $K 2$ and $K 6$ on hepatic steatosis and body weight in rats with HF/HF-induced non-alcoholic fatty liver disease (NAFLD). (a) Histochemical and (b) morphometric analysis of liver samples and (c) body weight changes in rats on $\mathrm{HF} / \mathrm{HF}$ diet with or without $\mathrm{K} 2$ and $\mathrm{K} 6$. NC, normal diet control; HC, HF/HF diet control; PC, HF/HF diet with silymarin; K2, HF/HF diet with ATG-K2; K6, $\mathrm{HF} / \mathrm{HF}$ diet with ATG-K6. The data are presented as mean $\pm \mathrm{SD}$. Means with different letters $(\mathrm{a}, \mathrm{b})$ are significantly different, $p<0.05$, whereas those with similar letters are not different. The groups were compared using one-way ANOVA followed by Duncan's multiple range tests.

Table 2. Body weight of rats in each groups (g).

\begin{tabular}{ccccc}
\hline Groups & $\begin{array}{c}\text { Initial Body } \\
\text { Weight }\end{array}$ & $\begin{array}{c}\text { Body Weight after } \\
\text { Randomization } \\
\text { (A) }\end{array}$ & $\begin{array}{c}\text { Terminal Body } \\
\text { Weight (B) }\end{array}$ & $\begin{array}{c}\text { Body Weight Gain } \\
\text { (B-A) }\end{array}$ \\
\hline NC & $194.0 \pm 8.4$ & $485.5 \pm 38.9^{\mathrm{a}}$ & $622.8 \pm 55.6^{\mathrm{a}}$ & $137.2 \pm 30.8^{\mathrm{a}}$ \\
$\mathrm{HC}$ & & $522.4 \pm 29.5^{\mathrm{b}}$ & $733.3 \pm 87.2^{\mathrm{b}}$ & $210.9 \pm 63.1^{\mathrm{b}}$ \\
PC & \multirow{2}{*}{$194.0 \pm 7.6$} & $522.4 \pm 28.6^{\mathrm{b}}$ & $680.4 \pm 57.5^{\mathrm{ab}}$ & $158.0 \pm 35.9^{\mathrm{a}}$ \\
K2 & & $521.9 \pm 31.1^{\mathrm{b}}$ & $677.9 \pm 64.8^{\mathrm{ab}}$ & $156.0 \pm 37.7^{\mathrm{a}}$ \\
K6 & & $523.6 \pm 27.1^{\mathrm{b}}$ & $697.1 \pm 55.6^{\mathrm{b}}$ & $173.5 \pm 30.9^{\mathrm{a}}$ \\
\hline
\end{tabular}

$\mathrm{NC}$, Normal diet control; HC, high fat/high fructose (HF/HF) diet control; PC, HF/HF diet with silymarin; K2, HF/HF diet with ATG-K2; K6, HF/HF diet with ATG-K6. The data are presented as mean \pm SD. Means with different letters $(\mathrm{a}, \mathrm{b})$ are significantly different, $p<0.05$, whereas those with similar letters are not different. The groups were compared using one-way ANOVA followed by Duncan's multiple range tests. 


\subsection{The effects of L. plantarum K2 and K6 on Serum Biochemical Parameters}

The activities of serum ALT, AST, and ALP were measured to evaluate the effect of K2 and K6 on liver function and damage. Serum ALT, AST, and ALP levels were significantly higher in the HC group than in the NC group (Figure 3a-c). Treatment with K2 and K6 significantly suppressed this effect, suggesting that both K2 and K6 have hepatoprotective properties. Levels of TG, TC, HDL-c, fasting serum glucose, adiponectin, and leptin were determined to assess the effects of K2 and K6 on serum lipid profile. As shown in Figure 4a-f, the HF/HF diet-induced increase in TG, TC, glucose, and leptin levels was significantly attenuated by treatment with $\mathrm{K} 2$ and K6. In addition, the decrease in HDL-c and adiponectin levels experienced by HC rats was prevented by administration of either strain. To assess the effect of $\mathrm{K} 2$ and $\mathrm{K} 6$ on antioxidant enzymes, we measured the activities of serum SOD, GPx, and CAT. The enzyme activities were markedly lower in the HC group than in the NC group; treatment with K2 and K6 suppressed this effect (Figure 4g-i).
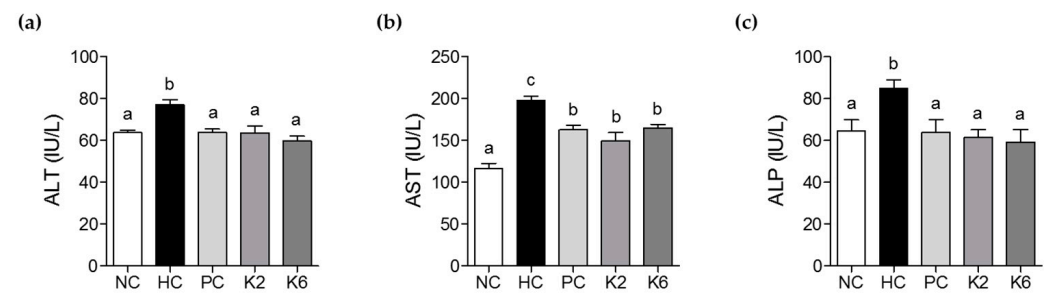

Figure 3. Effects of K2 and K6 on serum ALT, AST, and ALP levels in the rat model of HF/HF-induced NAFLD. (a) Serum ALT, (b) serum AST, and (c) serum ALP levels. NC, normal diet control; HC, HF/HF diet control; PC, HF/HF diet with silymarin; K2, HF/HF diet with ATG-K2; K6, HF/HF diet with ATG-K6; ALT, alanine aminotransferase; AST, aspartate aminotransferase; ALP, alkaline phosphatase. The data are presented as mean \pm SD. Means with different letters $(a, b$, and $c)$ are significantly different, $p<0.05$, whereas those with similar letters are not different. The groups were compared using one-way ANOVA followed by Duncan's multiple range tests.

(a)
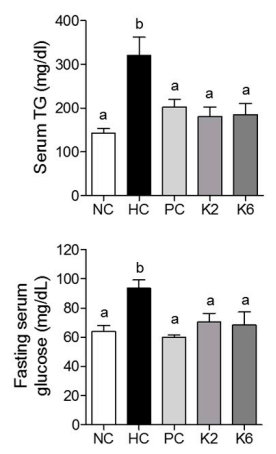

(g)

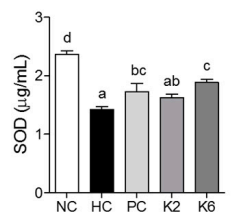

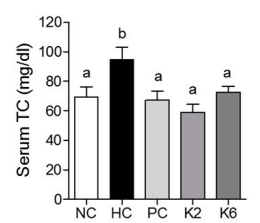

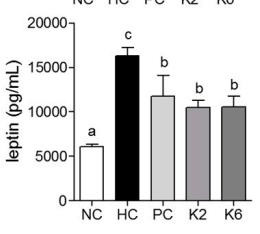

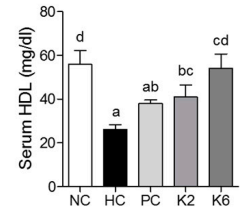

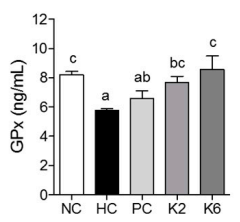

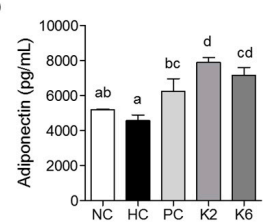

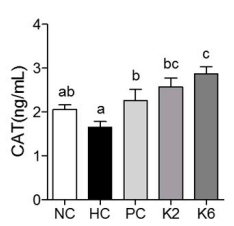

Figure 4. Effects of $\mathrm{K} 2$ and $\mathrm{K} 6$ on serum biochemical parameters in the rat model of HF/HF-induced NAFLD. (a) Serum TG, (b) serum TC, (c) serum HDL, (d) fasting serum glucose, (e) leptin, (f) adiponectin, (g) superoxide dismutase (SOD), (h) GPx, and (i) CAT levels. NC, normal diet control; HC, HF/HF diet control; PC, HF/HF diet with silymarin; K2, HF/HF diet with ATG-K2; K6, HF/HF diet with ATG-K6; TG, triglycerides; TC, total cholesterol; HDL, high-density lipoprotein; SOD, superoxide dismutase; GPx, glutathione peroxidase; CAT, catalase. The data are presented as mean $\pm \mathrm{SD}$. Means with different letters ( $a, b, c$, and d) are significantly different, $p<0.05$, whereas those with similar letters are not different. The groups were compared using one-way ANOVA followed by Duncan's multiple range tests. 


\subsection{The Effects of L. plantarum K2 and K6 on Hepatic Biochemical Parameters}

TG and TC levels were measured in rat liver tissue to determine the effect of L. plantarum K2 and K6 on HF/HF diet-induced NAFLD. Hepatic TG levels were lower in K2- and K6-treated rats compared to untreated rats. There was a significant difference in hepatic TG levels between K2 and PC groups, but not between $\mathrm{K} 2$ and $\mathrm{K} 6$ groups (Figure 5a). Hepatic TC and MDA levels were significantly higher in $\mathrm{HC}$ rats than in NC rats. In contrast, they were reduced in K2- and K6-treated rats (Figure 5b). Similarly, MDA levels were higher in the HC group than in the NC group; this effect was also suppressed by treatment with K2 or K6 (Figure 5c).

(a)

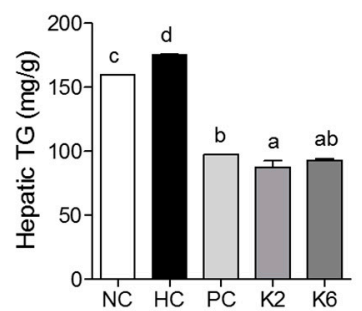

(b)

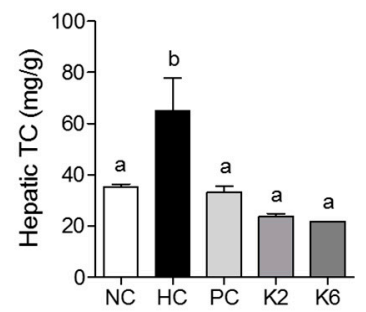

(c)

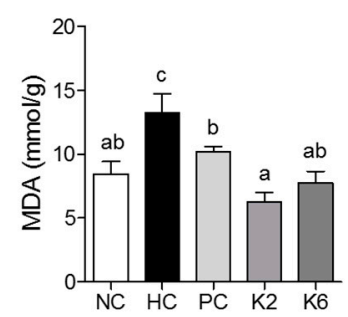

Figure 5. Effects of $\mathrm{K} 2$ and $\mathrm{K} 6$ on hepatic biochemical parameters in the rat model of HF/HF-induced NAFLD. (a) Hepatic TG, (b) TC, and (c) MDA levels. NC, normal diet control; HC, HF/HF diet control; PC, HF/HF diet with silymarin; K2, HF/HF diet with ATG-K2; K6, HF/HF diet with ATG-K6; TG, triglycerides; TC, total cholesterol; MDA, malondialdehyde. The data are presented as mean $\pm \mathrm{SD}$. Means with different letters ( $\mathrm{a}, \mathrm{b}, \mathrm{c}$, and d) are significantly different, $p<0.05$, whereas those with similar letters are not different. The groups were compared using one-way ANOVA followed by Duncan's multiple range tests.

\subsection{The Effects of L. plantarum K2 and K6 on Lipogenesis-Related Gene Expression in the Liver}

To investigate the molecular mechanisms underlying the effects of L. plantarum K2 and K6 on NAFLD, expression of lipogenesis-related genes was examined by RT-PCR and immunoblotting. SREBP-1c and FAS mRNA levels were significantly upregulated in the livers of $\mathrm{HC}$ rats compared to NC rats. mRNA expression of SREBP-1c and FAS was significantly lower in the K2 and K6 groups than in the HC group (Figure 6a,b). Similarly, mRNA levels of C/EBP were increased in the HC group, and tended to decrease in the PC and $\mathrm{K} 2$ groups. In line with this, immunoblot analysis showed that protein levels of SREBP-1c, FAS, and C/EBP were downregulated in K2 and K6 rats compared to HC rats (Figure $6 \mathrm{~d}-\mathrm{f}$ ). These results indicate that the therapeutic effects of L. plantarum $\mathrm{K} 2$ and $\mathrm{K} 6$ on NAFLD are associated with downregulation of lipogenesis. 
(a)

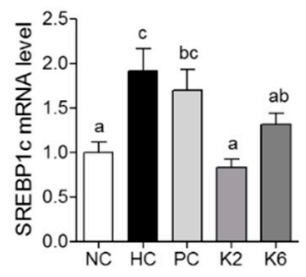

(d)

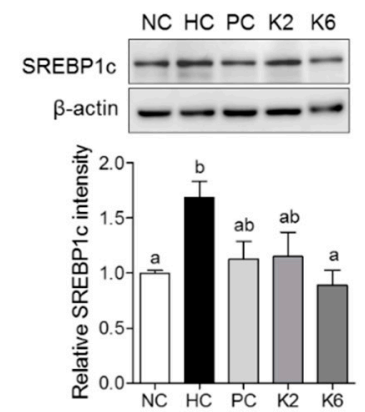

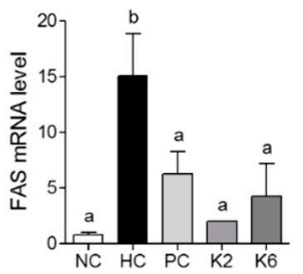

(e)
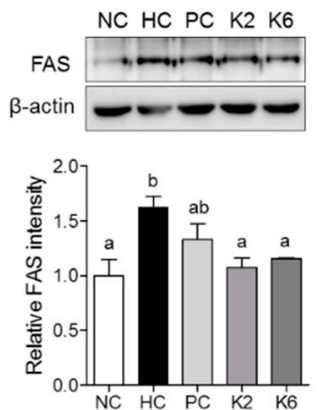

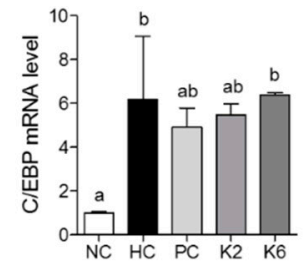

(f)

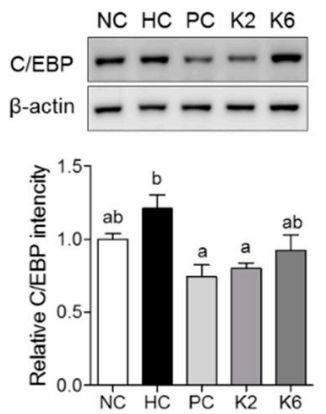

Figure 6. Effects of $\mathrm{K} 2$ and $\mathrm{K} 6$ on mRNA and protein expression of lipogenesis-related genes in the rat model of HF/HF-induced NAFLD. mRNA expression level of (a) SREBP-1c, (b) FAS, and (c) C/EBP in the liver. Western blot analysis of (d) SREBP-1c, (e) FAS, and (f) C/EBP expression in the liver. NC, normal diet control; HC, HF/HF diet control; PC, HF/HF diet with silymarin; K2, HF/HF diet with ATG-K2; K6, HF/HF diet with ATG-K6; SREBP-1c, sterol regulatory element binding protein 1c; FAS, fatty acid synthase; C/EBP, CCAAT/enhancer-binding protein. The data are presented as mean $\pm \mathrm{SD}$. Means with different letters $(a, b)$ are significantly different, $p<0.05$, whereas those with similar letters are not different. The groups were compared using one-way ANOVA followed by Duncan's multiple range tests.

\subsection{The Effects of L. plantarum K2 and K6 on Lipid Metabolism in the Liver}

To evaluate the effects of L. plantarum K2 and K6 on hepatic lipid metabolism, expression of genes involved in fatty acid oxidation, such as ACC and CPT-1, was measured by qRT-PCR. Expression of ACC mRNA was lower in K2- and K6- treated rats than in HC rats. There were no significant differences in ACC expression between the PC, K2, and K6 groups (Figure 7a). CPT-1 mRNA levels were also higher in the $\mathrm{K} 2$ and $\mathrm{K} 6$ groups than in the HC group (Figure $7 \mathrm{~b}$ ). To investigate whether $L$. plantarum K2 and K6 activates AMPK signaling, we performed an immunoblot analysis. As shown in Figure 7d-e, phosphorylation levels of both markers were enhanced following treatment with $\mathrm{K} 2$ or $\mathrm{K} 6$. CPT-1 protein expression was also higher in K2- and K6-treated than in in the HC group (Figure 7f). 
(a)

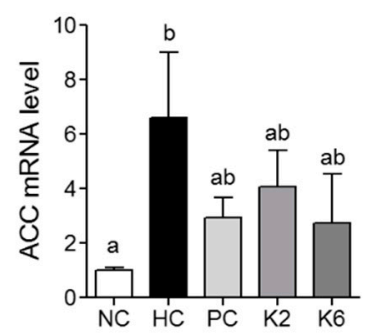

(c)
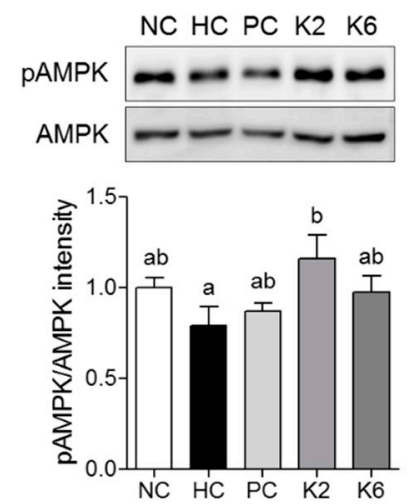

(b)

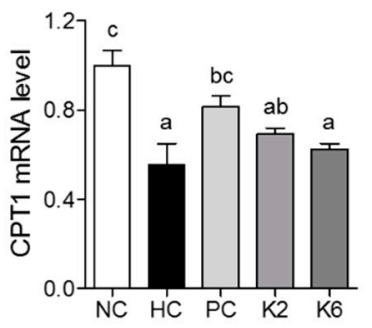

(d)
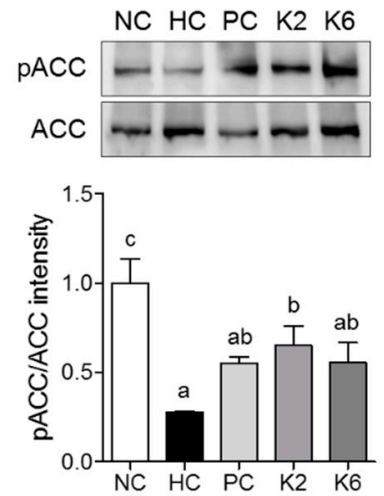

(e)
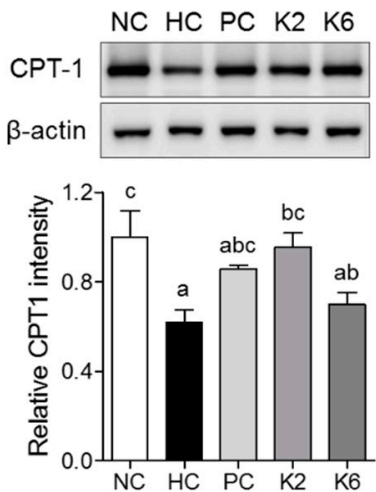

Figure 7. Effects of $\mathrm{K} 2$ and $\mathrm{K} 6$ on mRNA, protein, and phosphorylation levels of lipid metabolism-related markers in the rat model of HF/HF-induced NAFLD. mRNA expression levels of (a) ACC and (b) CPT-1 in the liver. Western blot analysis of (c) phosphorylated and total AMPK, (d) phosphorylated and total ACC, and (e) CPT-1 in the liver. Total AMPK and ACC were used as protein loading controls for phosphorylated AMPK and ACC, respectively. NC, normal diet control; HC, HF/HF diet control; PC, HF/HF diet with silymarin; K2, HF/HF diet with ATG-K2; K6, HF/HF diet with ATG-K6; AMPK, AMP-activated protein kinase; ACC, acetyl-CoA carboxylase; CPT-1, carnitine palmitoyltransferase-1. The data are presented as mean \pm SD. Means with different letters $(a, b$, and $c)$ are significantly different, $p<0.05$, whereas those with similar letters are not different. The groups were compared using one-way ANOVA followed by Duncan's multiple range tests.

\subsection{The Effects of L. plantarum Administration on Fecal Microbiota Composition}

To examine the effects of L. plantarum species on the gut microbial community, the bacterial composition of three pooled fecal samples from each experimental group was analyzed. The beta-diversity was examined through PCoA using the weighted UniFrac distance matrix. The microbiota of the HC group was clearly separated from those of other groups (Figure 8a). Mirroring the PCoA result, the microbiota of the PC, K2, and K6 groups had a relatively similar composition to that of the NC group, while the HC group showed a different bacterial community structure (Figure $8 \mathrm{~b}$ and Table S1) with a higher abundance of Firmicutes (90.5\%) and lower abundance of Bacteroidetes (5.6\%) than in the other groups. NC, PC, K2, and $\mathrm{K} 6$ rats displayed $67.2 \%-77.1 \%$ abundance of Firmicutes and $12.4 \%-25.8 \%$ abundance of Bacteroidetes. Significant differences in bacterial populations were confirmed using student's $t$-test (Figure 9). K2- and K6-treated rats showed similar changes in bacterial community structure as NC and PC rats. At the family level, Ruminococcaceae (phylum Firmicutes) and Lachnospiraceae (phylum Firmicutes) were more abundant, while Bacteroidaceae (phylum Bacteroidetes) and Bacteroidales S24-7 (phylum Bacteroidetes) were less abundant in the HC group than in the other groups (Figure 10). 
(a)

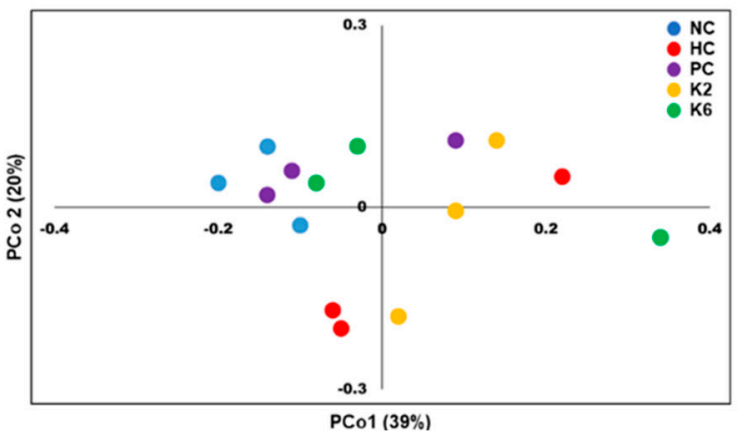

(b)

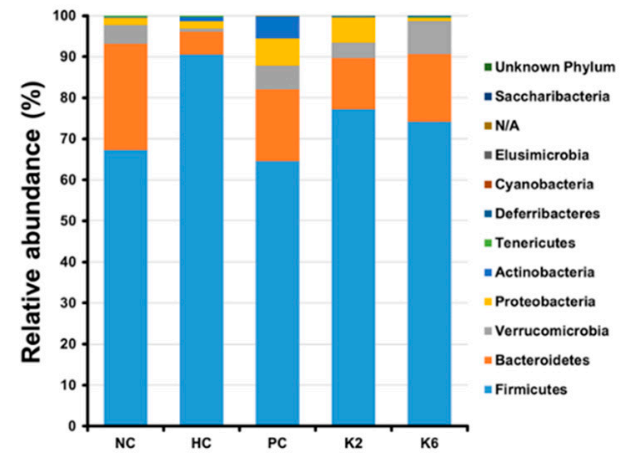

Figure 8. Fecal microbial diversity in each group. (a) Principal coordinates analysis (PCoA) plot using weighted UniFrac distance matrix; (b) Bar plot of relative bacterial phylum abundance. Each bar represents results of three pooled fecal samples. NC, normal diet control; HC, HF/HF diet control; PC, HF/HF diet with silymarin; K2, HF/HF diet with ATG-K2; K6, HF/HF diet with ATG-K6.

(a)

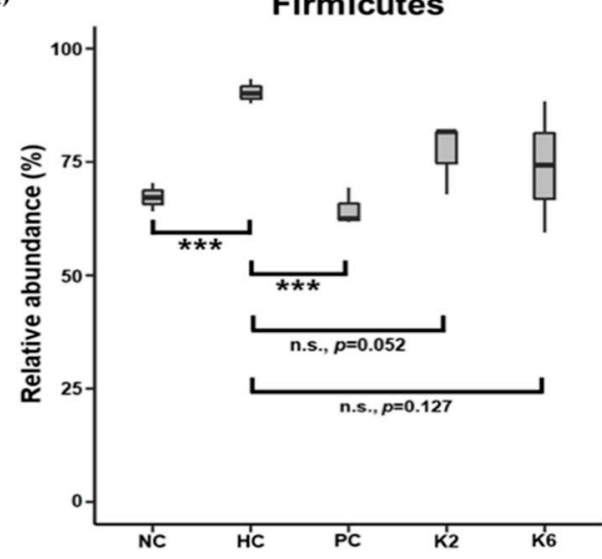

(b)

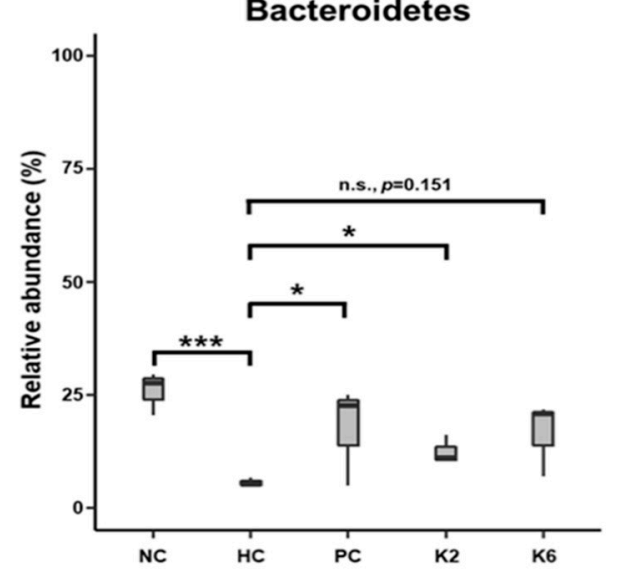

Figure 9. Relative abundance of phyla (a) Firmicutes and (b) Bacteroidetes in each group. Each box plot represents results of three pooled fecal samples. NC, normal diet control; HC, HF/HF diet control; PC, HF/HF diet with silymarin; K2, HF/HF diet with ATG-K2; K6, HF/HF diet with ATG-K6. ${ }^{*} p<0.05,{ }^{* * *} p<0.005$, n.s., not significant versus the HC group. Student's t-test was used to compare the group means.

Ruminococcaceae

Bacteroidales S24-7
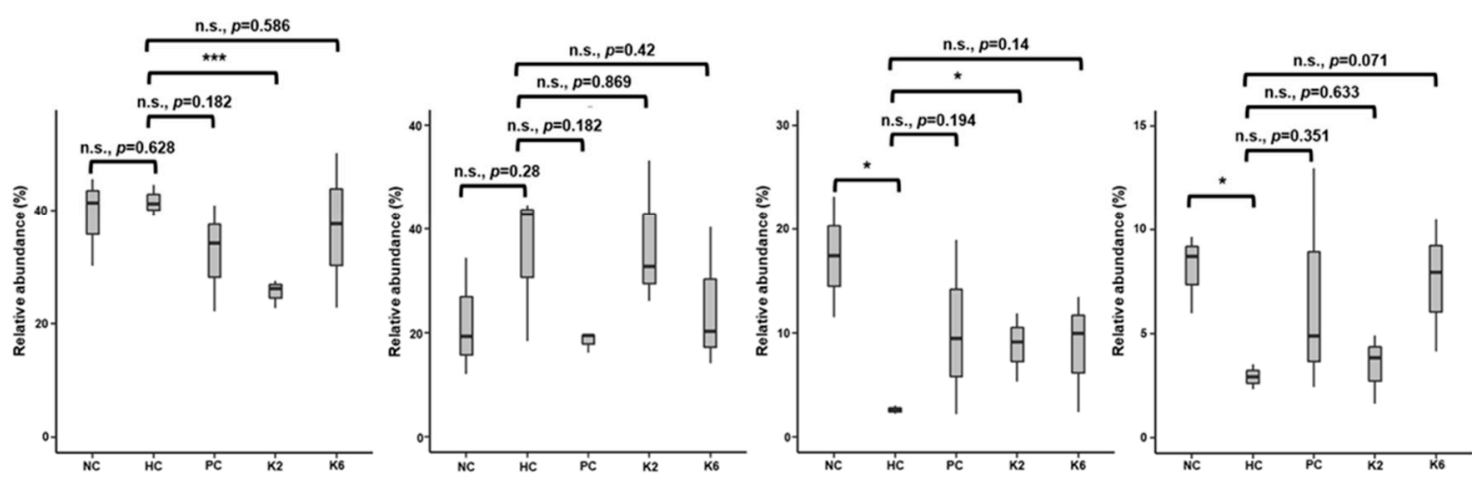

Figure 10. Relative abundance of family (a) Ruminococcaceae, (b) Lachnospiraceae, (c) Bacteroidaceae, and (d) Bacteroidales S24-7 in each group. Each box plot represents results of three pooled fecal samples. NC, normal diet control; HC, HF/HF diet control; PC, HF/HF diet with silymarin; K2, HF/HF diet with ATG-K2; K6, HF/HF diet with ATG-K6. ${ }^{*} p<0.05,{ }^{* * *} p<0.005$, n.s., not significant versus the HC group. Student's t-test was used to compare the group means. 


\section{Discussion}

Probiotics have been used traditionally for gastrointestinal health in the form of yogurt and others [27]. Most of the immune cells against disease are produced in the intestine, therefore there is a growing interest in intestinal health due to probiotic intake. This interest extends to the improvement of various inflammatory diseases. Particularly, probiotic treatment is an effective and safe approach to reversing metabolic abnormalities observed not only in obesity [28,29], but also in NAFLD [30]. In this study, treatment with two L. plantarum strains, K2 and K6, improved HF/HF diet-induced NAFLD in rats by regulating liver function, serum lipid levels, and lipogenesis via the AMPK pathway. Our literature investigation confirmed that several probiotics administered alone or in combination, have improvement effects on liver function in vivo [17,31]. L. plantarum NCU116, isolated from pickled vegetables, has indicated the effect amelioration of NAFLD by lowering the cholesterol property [17]. Another study also showed that cocktail of probiotics (L. acidophilus, L. plantarum, Bifidobacterium, and Bacillus subtilis strains) affected host adipose tissue hormone levels such as leptin, resistin, and hepatic function markers [31]. These results provide the validity of our results, suggesting that our results will contribute to discovering ameliorative action mechanisms of probiotics against NAFLD.

Measurement of liver function-related enzymes, such as ALT, AST, and ALP, can detect damage to the hepatocellular and biliary tracts [32]. Increased activity of ALT and AST can indicate NAFLD [33]. In particular, ALT is a hepatocyte-specific enzyme whose elevated serum levels directly reflect liver damage [32,34]. Our results showed that serum ALT levels were high in rats in the HC group, and low in rats in the K2- and K6-treated groups. In a previous study, L. paracasei Jlus66 isolated from natural fermented milk attenuated serum ALT activity in a rat model of high fat diet-induced NAFLD [30]. Taken together, these results suggest that probiotics isolated from fermented foods could exert protective effects on NAFLD by reducing ALT activity.

Increased free radical load in NAFLD can affect $\beta$-oxidation to enhance the production of reactive oxygen species (ROS) [35]. Accumulation of ROS causes oxidative stress, resulting in protein carbonylation and DNA damage [36]. MDA is produced by lipid peroxidation and considered a marker oxidative stress. In addition, SOD acts as a defender of oxidative stress that changes oxygen radicals to hydrogen peroxide and dioxygen [37]. In this study, the enzyme activity of SOD, GPx, and CAT was measured in the serum to assess the antioxidant effects of K2 and K6. Both K2- and K6-treated rats displayed higher antioxidant activities than HC rats. MDA levels were decreased in the K2 and K6 group, these results were similar to other study using L. plantarum NA 136 [16]. Our results demonstrate that $\mathrm{K} 2$ and $\mathrm{K} 6$ have the potential to be developed as hepatoprotective agents as indicated by higher antioxidant activities on the NAFLD rat model.

Nrf2 plays a crucial role in cellular defenses against oxidative stress by regulating expression of antioxidant enzymes [38]. Normally, Keap1 binds Nrf2 to sequester it in the cytoplasm. Once cells are exposed to the oxidative stress, Nrf2 is released from Keap1 and translocates into the nucleus. Once in the nucleus, Nrf2 dimerizes with Maf and stimulates the expression of antioxidant stress genes [39]. A previous study showed that CAT promotes Nrf2 translocation and targets its downstream genes, such as HO-1 gene, to trigger the Nrf2 defense system [40]. Reportedly, treatment with L. plantarum NA136 increased CAT expression, which was attenuated in the HFD/F group [16]. This in turn triggers Nrf2 translocation to regulate expression of cellular antioxidant enzymes, including SOD and HO-1. Bacillus amyloliquefaciens SC06 has also been reported to increase the level of CAT and activate the Nrf2/Keap1 signaling pathway to boost cellular antioxidant status [41]. Further studies are required to confirm the involvement of Nrf2/Keap1 signaling in L. plantarum K2- and K6-induced effects.

NAFLD is closely related to insulin resistance in the liver [42]. Excessive glucose levels and insulin release in $\mathrm{HF} / \mathrm{HF}$ diet can promote DNL via hepatic transcription factors, such as SREBP-1. Activated by downstream signaling, SREBP-1 increases FAS expression [43], which is involved in fatty acid synthesis. In NAFLD, FAS stimulates fatty acid accumulation in hepatocytes. In this study, lipid metabolism-associated pathways, such as leptin, adiponectin, SREBP-1, and ACC signaling, were restored by L. plantarum $\mathrm{K} 2$ and K6, which in turn decreased accumulation of TG and TC in the serum, 
resulting in weight loss. Adipose tissue can release free fatty acids to the liver, where the levels of leptin, adiponectin are altered, decreasing cholesterol levels in the blood and dampening anti-inflammatory processes mediated by cytokines such as TNF- $\alpha$ and IL-6 [44]. The results of the present study, and of our previous investigation of anti-inflammatory effects of L. plantarum K2 and K6 [18] suggest that the therapeutic effects of probiotics are mediated by regulation of insulin-, inflammation-, and lipogenesis-associated pathways. Moreover, these changes in serum lipid biomarkers by K2 and K6 are thought to also be effective in reducing the fat accumulation, similar to the function of probiotics such as L. gasseri BNR17 [45].

Oral administration of L. plantarum strains may alter intestinal and fecal microbiota [46]. Treatment with L. plantarum $\mathrm{K} 2$ or $\mathrm{K} 6$ induced similar changes to the composition of fecal microbiome. Although not all results reached statistical significance, administration of either $\mathrm{K} 2$ or $\mathrm{K} 6$ induced a shift in the microbial community that resulted in an increased abundance of Bacteroidetes, (including family Bacteroidaceae and Bacteroidales S24-7) and decreased abundance of Firmicutes, with respect to the untreated rats. Further studies are required to identify the active substances of K2 and K6 strains, modulation of Bacteroidetes population through oral administration of Lactobacillus, links to progression of fatty liver and NAFLD [47,48]. Interestingly, fecal microbiota of the silymarin-treated rats (positive control) also showed an increased proportion of Bacteroidetes and decreased proportion of Firmicutes. High relative abundance of Bacteroidetes in the gut microbiota correlates with liver health [49]; thus, $L$. plantarum $\mathrm{K} 2$ and $\mathrm{K} 6$ are potential probiotics owing to their ability to boost Bacteroidetes levels.

\section{Conclusions}

In the present study, L. plantarum strains K2 and K6 ameliorated HF/HF diet-induced NAFLD by regulating liver function and expression of antioxidant enzymes and lipogenesis-related genes. In addition, both strains were able to improve the composition of fecal microbiota. Our results provide a basis for the development of novel therapeutic strategies for NAFLD.

Supplementary Materials: The following are available online at http://www.mdpi.com/2072-6643/12/2/542/s1, Table S1. ANI values for Lactobacillus plantarum strains. Tables S2-S4. Fecal Microbial Community Structure results.

Author Contributions: E.-J.P. and H.-J.L. designed the study; Y.H.L. and D.Y.J. prepared the bacterial strains; G.-S.P. performed microbial community analysis; J.K. project administration; E.-J.P., Y.-S.L., and S.M.K. performed the experiments; E.-J.P. analyzed the data; E.-J.P., Y.-S.L., and G.S.P. wrote the manuscript; H.-J.L. reviewed the manuscript. All authors have read and agreed to the published version of the manuscript.

Funding: This work was supported by “Food Functionality Evaluation Program” under the Ministry of Agriculture, Food and Rural Affairs and partly Korea Food Research Institute.

Acknowledgments: We thank Jong Yeon Kim for technical assistance.

Conflicts of Interest: The authors declare no conflict of interest.

\section{References}

1. Samuel, V.T.; Shulman, G.I. Nonalcoholic Fatty Liver Disease as a Nexus of Metabolic and Hepatic Diseases. Cell Metab. 2018, 27, 22-41. [CrossRef]

2. Machado, M.; Marques-Vidal, P.; Cortez-Pinto, H. Hepatic histology in obese patients undergoing bariatric surgery. J. Hepatol. 2006, 45, 600-606. [CrossRef]

3. Cobbina, E.; Akhlaghi, F. Non-alcoholic fatty liver disease (NAFLD) - pathogenesis, classification, and effect on drug metabolizing enzymes and transporters. Drug Metab. Rev. 2017, 49, 197-211. [CrossRef]

4. Bedossa, P. Pathology of non-alcoholic fatty liver disease. Liver Int. Off. J. Int. Assoc. Study Liver 2017, 37, 85-89. [CrossRef]

5. Adams, L.A.; Lymp, J.F.; St Sauver, J.; Sanderson, S.O.; Lindor, K.D.; Feldstein, A.; Angulo, P. The natural history of nonalcoholic fatty liver disease: A population-based cohort study. Gastroenterology 2005, 129, 113-121. [CrossRef]

6. Ter Horst, K.W.; Serlie, M.J. Fructose Consumption, Lipogenesis, and Non-Alcoholic Fatty Liver Disease. Nutrients 2017, 981. [CrossRef] 
7. Herman, M.A.; Samuel, V.T. The Sweet Path to Metabolic Demise: Fructose and Lipid Synthesis. Trends Endocrinol. Metab. 2016, 27, 719-730. [CrossRef] [PubMed]

8. Softic, S.; Cohen, D.E.; Kahn, C.R. Role of Dietary Fructose and Hepatic De Novo Lipogenesis in Fatty Liver Disease. Dig. Dis. Sci. 2016, 61, 1282-1293. [CrossRef] [PubMed]

9. Jensen, T.; Abdelmalek, M.F.; Sullivan, S.; Nadeau, K.J.; Green, M.; Roncal, C.; Nakagawa, T.; Kuwabara, M.; Sato, Y.; Kang, D.H.; et al. Fructose and sugar: A major mediator of non-alcoholic fatty liver disease. J. Hepatol. 2018, 68, 1063-1075. [CrossRef] [PubMed]

10. Salomone, F.; Godos, J.; Zelber-Sagi, S. Natural antioxidants for non-alcoholic fatty liver disease: Molecular targets and clinical perspectives. Liver Int. 2016, 36, 5-20. [CrossRef]

11. Cacciapuoti, F.; Scognamiglio, A.; Palumbo, R.; Forte, R.; Cacciapuoti, F. Silymarin in non alcoholic fatty liver disease. World J. Hepatol. 2013, 5, 109-113. [CrossRef] [PubMed]

12. Xiao, M.W.; Lin, S.X.; Shen, Z.H.; Luo, W.W.; Wang, X.Y. Systematic Review with Meta-Analysis: The Effects of Probiotics in Nonalcoholic Fatty Liver Disease. Gastroenterol. Res. Pract. 2019, 2019, 1484598. [CrossRef] [PubMed]

13. Aller, R.; De Luis, D.A.; Izaola, O.; Conde, R.; Gonzalez Sagrado, M.; Primo, D.; De La Fuente, B.; Gonzalez, J. Effect of a probiotic on liver aminotransferases in nonalcoholic fatty liver disease patients: A double blind randomized clinical trial. Eur. Rev. Med. Pharm. Sci. 2011, 15, 1090-1095.

14. Famouri, F.; Shariat, Z.; Hashemipour, M.; Keikha, M.; Kelishadi, R. Effects of Probiotics on Nonalcoholic Fatty Liver Disease in Obese Children and Adolescents. J. Pediatr. Gastroenterol. Nutr. 2017, 64, 413-417. [CrossRef]

15. Seddik, H.A.; Bendali, F.; Gancel, F.; Fliss, I.; Spano, G.; Drider, D. Lactobacillus plantarum and Its Probiotic and Food Potentialities. Probiotics Antimicrob. Proteins 2017, 9, 111-122. [CrossRef]

16. Zhao, Z.; Wang, C.; Zhang, L.; Zhao, Y.; Duan, C.; Zhang, X.; Gao, L.; Li, S. Lactobacillus plantarum NA136 improves the non-alcoholic fatty liver disease by modulating the AMPK/Nrf2 pathway. Appl. Microbiol. Biotechnol. 2019, 103, 5843-5850. [CrossRef]

17. Li, C.; Nie, S.P.; Zhu, K.X.; Ding, Q.; Li, C.; Xiong, T.; Xie, M.Y. Lactobacillus plantarum NCU116 improves liver function, oxidative stress and lipid metabolism in rats with high fat diet induced non-alcoholic fatty liver disease. Food Funct. 2014, 5, 3216-3223. [CrossRef]

18. Beck, B.R.; Park, G.S.; Lee, Y.H.; Im, S.; Jeong, D.Y.; Kang, J. Whole Genome Analysis of Lactobacillus plantarum Strains Isolated From Kimchi and Determination of Probiotic Properties to Treat Mucosal Infections by Candida albicans and Gardnerella vaginalis. Front. Microbiol. 2019, 10, 433. [CrossRef]

19. Burton, D.T.; Cooper, K.R.; Goodfellow, W.L., Jr.; Rosenblatt, D.H. Uptake, elimination, and metabolism of 14C-picric acid and 14C-picramic acid in the American oyster (Crassostrea virginica). Arch. Environ. Contam. Toxicol. 1984, 13, 653-663. [CrossRef]

20. Lin, M.Y.; Yen, C.L. Antioxidative ability of lactic acid bacteria. J. Agric. Food Chem. 1999, 47, 1460-1466. [CrossRef]

21. Yoon, S.H.; Ha, S.M.; Lim, J.; Kwon, S.; Chun, J. A large-scale evaluation of algorithms to calculate average nucleotide identity. Antonie Van Leeuwenhoek 2017, 110, 1281-1286. [CrossRef]

22. Park, M.; Yoo, J.H.; Lee, Y.S.; Lee, H.J. Lonicera caerulea Extract Attenuates Non-Alcoholic Fatty Liver Disease in Free Fatty Acid-Induced HepG2 Hepatocytes and in High Fat Diet-Fed Mice. Nutrients 2019, 11, 494. [CrossRef] [PubMed]

23. Caporaso, J.G.; Lauber, C.L.; Walters, W.A.; Berg-Lyons, D.; Huntley, J.; Fierer, N.; Owens, S.M.; Betley, J.; Fraser, L.; Bauer, M. Ultra-high-throughput microbial community analysis on the Illumina HiSeq and MiSeq platforms. ISME J. 2012, 6, 1621. [CrossRef] [PubMed]

24. Caporaso, J.G.; Kuczynski, J.; Stombaugh, J.; Bittinger, K.; Bushman, F.D.; Costello, E.K.; Fierer, N.; Pena, A.G.; Goodrich, J.K.; Gordon, J.I. QIIME allows analysis of high-throughput community sequencing data. Nat. Methods 2010, 7, 335. [CrossRef]

25. Yilmaz, P.; Parfrey, L.W.; Yarza, P.; Gerken, J.; Pruesse, E.; Quast, C.; Schweer, T.; Peplies, J.; Ludwig, W.; Glöckner, F.O. The SILVA and "all-species living tree project (LTP)" taxonomic frameworks. Nucleic Acids Res. 2013, 42, D643-D648. [CrossRef] [PubMed]

26. Lozupone, C.; Lladser, M.E.; Knights, D.; Stombaugh, J.; Knight, R. UniFrac: An effective distance metric for microbial community comparison. ISME J. 2011, 5, 169. [CrossRef] 
27. Rivera-Espinoza, Y.; Gallardo-Navarro, Y. Non-dairy probiotic products. Food Microbiol. 2010, $27,1-11$. [CrossRef]

28. Park, S.S.; Lee, Y.J.; Kang, H.; Yang, G.; Hong, E.J.; Lim, J.Y.; Oh, S.; Kim, E. Lactobacillus amylovorus KU4 ameliorates diet-induced obesity in mice by promoting adipose browning through PPARgamma signaling. Sci. Rep. 2019, 9, 20152. [CrossRef]

29. John, G.K.; Mullin, G.E. The Gut Microbiome and Obesity. Curr. Oncol. Rep. 2016, 18, 45. [CrossRef]

30. Ye, H.; Li, Q.; Zhang, Z.; Sun, M.; Zhao, C.; Zhang, T. Effect of a novel potential probiotic Lactobacillus paracasei Jlus66 isolated from fermented milk on nonalcoholic fatty liver in rats. Food Funct. 2017, 8, 4539-4546. [CrossRef]

31. Al-Muzafar, H.M.; Amin, K.A. Probiotic mixture improves fatty liver disease by virtue of its action on lipid profiles, leptin, and inflammatory biomarkers. BMC Complement. Altern. Med. 2017, 17, 43. [CrossRef]

32. Oh, R.C.; Hustead, T.R.; Ali, S.M.; Pantsari, M.W. Mildly Elevated Liver Transaminase Levels: Causes and Evaluation. Am. Fam. Physician 2017, 96, 709-715.

33. Fraser, A.; Longnecker, M.P.; Lawlor, D.A. Prevalence of elevated alanine aminotransferase among US adolescents and associated factors: NHANES 1999-2004. Gastroenterology 2007, 133, 1814-1820. [CrossRef]

34. Liu, Z.; Que, S.; Xu, J.; Peng, T. Alanine aminotransferase-old biomarker and new concept: A review. Int. J. Med. Sci. 2014, 11, 925-935. [CrossRef]

35. Masarone, M.; Rosato, V.; Dallio, M.; Gravina, A.G.; Aglitti, A.; Loguercio, C.; Federico, A.; Persico, M. Role of Oxidative Stress in Pathophysiology of Nonalcoholic Fatty Liver Disease. Oxidative Med. Cell. Longev. 2018, 2018, 9547613. [CrossRef] [PubMed]

36. Tan, B.L.; Norhaizan, M.E.; Liew, W.P.; Sulaiman Rahman, H. Antioxidant and Oxidative Stress: A Mutual Interplay in Age-Related Diseases. Front. Pharmacol. 2018, 9, 1162. [CrossRef]

37. Padurariu, M.; Ciobica, A.; Hritcu, L.; Stoica, B.; Bild, W.; Stefanescu, C. Changes of some oxidative stress markers in the serum of patients with mild cognitive impairment and Alzheimer's disease. Neurosci. Lett. 2010, 469, 6-10. [CrossRef] [PubMed]

38. Chen, M.; Jiang, L.; Li, Y.; Bai, G.; Zhao, J.; Zhang, M.; Zhang, J. Hydrogen protects against liver injury during CO2 pneumoperitoneum in rats. Oncotarget 2018, 9, 2631-2645. [CrossRef] [PubMed]

39. Osburn, W.O.; Kensler, T.W. Nrf2 signaling: An adaptive response pathway for protection against environmental toxic insults. Mutat. Res. 2008, 659, 31-39. [CrossRef] [PubMed]

40. Chang, J.; Ma, J.Z.; Zeng, Q.; Cechova, S.; Gantz, A.; Nievergelt, C.; O'Connor, D.; Lipkowitz, M.; Le, T.H. Loss of GSTM1, a NRF2 target, is associated with accelerated progression of hypertensive kidney disease in the African American Study of Kidney Disease (AASK). Am. J. Physiol. Ren. Physiol. 2013, 304, F348-F355. [CrossRef]

41. Wang, Y.; Wu, Y.; Wang, Y.; Fu, A.; Gong, L.; Li, W.; Li, Y. Bacillus amyloliquefaciens SC06 alleviates the oxidative stress of IPEC-1 via modulating Nrf2/Keap1 signaling pathway and decreasing ROS production. Appl. Microbiol. Biotechnol. 2017, 101, 3015-3026. [CrossRef]

42. Bogdanova, K.; Poczatkova, H.; Uherkova, L.; Riegrova, D.; Rypka, M.; Feher, J.; Marchesini, G.; Vesely, J. Non-alcoholic fatty liver disease (NAFLD)-a novel common aspect of the metabolic syndrome. Biomed. Pap. Med. Fac. Univ. PalackyOlomoucCzechoslov. 2006, 150, 101-104. [CrossRef]

43. Jeon, T.I.; Osborne, T.F. SREBPs: Metabolic integrators in physiology and metabolism. Trends Endocrinol. Metab. 2012, 23, 65-72. [CrossRef]

44. Wong, C.P.; Kaneda, T.; Morita, H. Plant natural products as an anti-lipid droplets accumulation agent. J. Nat. Med. 2014, 68, 253-266. [CrossRef]

45. Kang, J.H.; Yun, S.I.; Park, M.H.; Park, J.H.; Jeong, S.Y.; Park, H.O. Anti-obesity effect of Lactobacillus gasseri BNR17 in high-sucrose diet-induced obese mice. PLoS ONE 2013, 8, e54617. [CrossRef] [PubMed]

46. Xie, Q.; Pan, M.; Huang, R.; Tian, X.; Tao, X.; Shah, N.P.; Wei, H.; Wan, C. Modulation of the small intestinal microbial community composition over short-term or long-term administration with Lactobacillus plantarum ZDY2013. J. Dairy Sci. 2016, 99, 6913-6921. [CrossRef] [PubMed]

47. Arslan, N. Obesity, fatty liver disease and intestinal microbiota. World J. Gastroenterol. WJG 2014, 20, 16452. [CrossRef] [PubMed] 
48. Ritze, Y.; Bárdos, G.; Claus, A.; Ehrmann, V.; Bergheim, I.; Schwiertz, A.; Bischoff, S.C. Lactobacillus rhamnosus GG protects against non-alcoholic fatty liver disease in mice. PLoS ONE 2014, 9, e80169. [CrossRef] [PubMed]

49. Mouzaki, M.; Comelli, E.M.; Arendt, B.M.; Bonengel, J.; Fung, S.K.; Fischer, S.E.; McGilvray, I.D.; Allard, J.P. Intestinal microbiota in patients with nonalcoholic fatty liver disease. Hepatology 2013, 58, 120-127. [CrossRef]

(C) 2020 by the authors. Licensee MDPI, Basel, Switzerland. This article is an open access article distributed under the terms and conditions of the Creative Commons Attribution (CC BY) license (http://creativecommons.org/licenses/by/4.0/). 